\title{
Affine Illumination Compensation for Multispectral Images
}

\author{
Pedro Latorre Carmona ${ }^{1}$, Reiner Lenz ${ }^{2}$, Filiberto $\mathrm{Pla}^{1}$, and Jose M. Sotoca ${ }^{1}$ \\ ${ }^{1}$ Depto. Lenguajes y Sistemas Informáticos, Universidad Jaume I \\ Campus del Riu Sec s/n, 12071, Castellón de la Plana, Spain \\ \{latorre,pla, sotoca\}@lsi.uji.es \\ ${ }^{2}$ Department of Science and Technology, Linköping University, Campus Norrköping \\ Norrköping, Sweden \\ reile@itn.liu.se
}

\begin{abstract}
We apply a general form of affine transformation model to compensate illumination variations in a series of multispectral images of a static scene and compare it to a particular affine and a diagonal transformation models. These models operate in the original multispectral space or in a lower-dimensional space obtained by Singular Value Decomposition (SVD) of the set of images. We use a system consisting of a multispectral camera and a light dome that allows the measurement of multispectral data under carefully controlled illumination conditions to generate a series of multispectral images of a static scene under varying illumination conditions. We evaluate the compensation performance using the CIELAB colour difference between images. The experiments show that the first 2 models perform satisfactorily in the original and lower dimensional spaces.
\end{abstract}

\section{Introduction}

Colour image processing based on data acquired by a camera follows a complex image formation process involving the properties of the camera, the reflection properties of the object points, the spectral characteristics of the illumination source and the geometric relation between all these components. In many applications we are however only interested in one of the parts of this process. For instance, in industrial inspection, remote sensing, or automatic colour correction, just to cite a few.

In this paper we propose the application of a general affine transformation model and analyze its performance in relation to other 2 models for the description of illumination changes. For this purpose we measure a static scene by a multichannel camera with 33 channels in the visible range of the spectrum. The scene illumination is provided by 120 lamps arranged on a semi-dome to provide a homogeneous illumination environment. The simplest model to describe color changes is given by a diagonal transform of the colour space [145]. This model, which corresponds to the so-called von-Kries adaptation in human colour vision [16], may be generalized by the introduction of an offset in the transformation model (a translation vector) without changing the diagonal nature of the transformation matrix [6], or by considering a particular class of affine transformations of the distribution of the colour content in the colour or spectral space [7]12]. In the cases where these affine models provide sufficiently accurate descriptions

B.K. Ersbøll and K.S. Pedersen (Eds.): SCIA 2007, LNCS 4522, pp. 522-531 2007.

(C) Springer-Verlag Berlin Heidelberg 2007 
of the colour changes they can be used to develop invariant features. Such features can be computed from $R G B$ images obtained by conventional commercial cameras, and from multispectral images. Typical application areas are illumination-invariant recognition of objects, or robust content-based retrieval of satellite images obtained under different illumination conditions and acquisition geometry [7|8|9]. In this paper we will analyze the applicability of a general affine transformation model with the help of series of multispectral images of static scenes under carefully controlled illumination changes.

The organization of the paper is as follows: in Section 2 we introduce the mathematical framework for the estimation of the parameters of the models. In Section 3 we describe the experimental device. In Section 4 we analyze the main features of the illumination changes, and compare the compensation models, both in the original and in a lower-dimensional spaces. Conclusions can be found in Section 5

\section{Affine Transformation Estimation for Illumination Changes}

We use a vector $x \in \mathbb{R}_{+}^{D}$ to denote a measurement from a $D$ bands multispectral camera of an object point under some illumination condition. Under a change in the illumination characteristics this vector will undergo a change which can be described by the transformation $x \rightarrow \widetilde{x}$. Assuming a specific model of light-camera interaction, Healey et al [7|8[9] consider the following Equation to describe this change:

$$
\widetilde{x}=\mathbf{A} \cdot x,
$$

where $\mathbf{A}$ is a $D \times D$ matrix. On the other hand, a general affine transformation model in the form of Eq. 2] [2] could be motivated by the inclusion of effects like noise in the camera, or others:

$$
\widetilde{x}=\mathbf{B} \cdot x+t,
$$

where $\mathbf{B}$ is also a $D \times D$ matrix. The estimation of the $\mathbf{B}$ matrix and the $t$ vector follows the description by Heikkila et al in [10], who applied the model to the movement of rigid objects in grey-scale images. We consider the two point sets $\mathbf{X}$ and $\widetilde{\mathbf{X}}$ as $N \times D$ matrices with $N$ the number of points in the set, and $\mathbf{C}$ and $\widetilde{\mathbf{C}}$ their covariance matrices. Let us introduce the Cholesky Factorization:

$$
\begin{aligned}
& \mathbf{C}=\mathbf{F} \cdot \mathbf{F}^{t} \\
& \widetilde{\mathbf{C}}=\widetilde{\mathbf{F}} \cdot \widetilde{\mathbf{F}}^{t}
\end{aligned}
$$

where $\mathbf{F}^{t}$ and $\widetilde{\mathbf{F}}^{t}$ are the transpose matrices of $\mathbf{F}$ and $\widetilde{\mathbf{F}}$ respectively. Points in the set are first whitened (only shown for the first group), i. e.

$$
y=\mathbf{F}^{-1} \cdot \bar{x}
$$

where $\bar{x}=x-\mathbb{E}\{x\}$. Taking into account Eq. 2 and Eq. 4 we have $\widetilde{\mathbf{F}} \cdot \widetilde{y}=\mathbf{B} \cdot \mathbf{F} \cdot y$, and creating a quadratic form of this last expression, we get

$$
\widetilde{\mathbf{F}} \cdot \widetilde{\mathbf{F}}^{t}=\mathbf{B} \cdot \mathbf{F} \cdot \mathbf{F}^{t} \cdot \mathbf{B}^{t}
$$


In [15] Sprinzak et al proved that an equation of the form $\mathbf{T} \cdot \mathbf{T}^{t}=\mathbf{S} \cdot \mathbf{S}^{t}$ has a solution of the form $\mathbf{T}=\mathbf{S} \cdot \mathbf{M}$, where $\mathbf{M}$ is an orthonormal matrix. This will help find the final relation $y \rightarrow \widetilde{y}$. Applying this to Eq. 5 and solving for $\mathbf{B}$, we have

$$
\mathbf{B}=\widetilde{\mathbf{F}} \cdot \mathbf{M}^{t} \cdot \mathbf{F}^{-1},
$$

Substituting Eq. 6]in $\widetilde{\mathbf{F}} \cdot \widetilde{y}=\mathbf{B} \cdot \mathbf{F} \cdot y$, yields:

$$
\widetilde{y}=\mathbf{M}^{t} \cdot y,
$$

The assessment of the $\mathbf{M}$ matrix is known as the Orthogonal Procrustes problem (see [13] for details). The solution matrix is $\mathbf{M}=\mathbf{V} \cdot \mathbf{W}^{t}$, where $\mathbf{V} \cdot \mathbf{D} \cdot \mathbf{W}^{t}$ is the Singular Value Decomposition of $\left(\mathbf{Y}^{t} \cdot \widetilde{\mathbf{Y}}\right)$. $\mathbf{Y}$ and $\widetilde{\mathbf{Y}}$ are $N \times D$ matrices formed by the vectors $y$ and $\widetilde{y}$ of the point sets. We obtain $\mathbf{B}$ replacing $\mathbf{M}$ in Eq. 6. Applying the Expectation Operator to $\widetilde{x}=\mathbf{B} \cdot x+t$, we get $t=\mathbb{E}\{\widetilde{x}\}-\mathbf{B} \cdot \mathbb{E}\{x\}$.

The matrix $\mathbf{A}$ in the particular affine transformation model can also be obtained using the definition of the Moore-Penrose inverse. Following [4], who applied this model to illumination changes in $R G B$ images, consider a $D \times N$ matrix $\mathbf{X}^{t}$ of points under some reference illumination condition. Denote by $\widetilde{\mathbf{X}^{t}}$ the corresponding matrix when there is an illumination change. The matrix $\mathbf{A}$ that accomplishes:

$$
\widetilde{\mathbf{X}^{t}} \approx \mathbf{A} \cdot \mathbf{X}^{t}
$$

is:

$$
\mathbf{A}=\widetilde{\mathbf{X}^{t}} \cdot\left[\mathbf{X}^{t}\right]^{+},
$$

$\left[\mathbf{X}^{t}\right]^{+}$is the Moore-Penrose inverse of matrix $\mathbf{X}^{t}$ (i. e., $\left.\left[\mathbf{X}^{t}\right]^{+}=\mathbf{X}\left(\mathbf{X}^{t} \cdot \mathbf{X}\right)^{-1}\right)$. The diagonal transform matrix $\mathbf{A}^{d}$ can be obtained from Eq. 9 Considering [4]:

$$
A_{i i}^{d}=\tilde{X}_{i}^{t} \cdot\left[X_{i}^{t}\right]^{+}=\frac{\tilde{X}_{i}^{t} \cdot X_{i}}{X_{i}^{t} \cdot X_{i}},
$$

where the single subscript $i$ denotes the $i$ th matrix row and the double subscript $i i$ denotes matrix element at row $i$ column $i$.

\section{Experimental Set-Up}

In our experiments we use a multichannel camera built around the CCD QImaging Retiga EX camera (12-bit, Monochrome Cooled camera without IR Filter). The sensor resolution is $1036 \times 1360$, down $516 \times 676$ pixels. Connected to the camera is a Liquid Crystal Tunable Filter (LCTF). The spectral sampling is $10 \mathrm{~nm}$ in the range from 400 to $720 \mathrm{~nm}$, resulting in 33 channels. The tunable filter is fixed in front of the camera and the camera/filter combination is mounted on top of the hemisphere, looking down towards its center.

The illumination chamber is shaped as a hemisphere with a diameter of $60 \mathrm{~cm}$ and contains 120 halogen lights of 10 Watts each. The lamps are powered by three-phase AC 12 volts adjustable power supply. Each group powers 40 lamps. The even spatial 


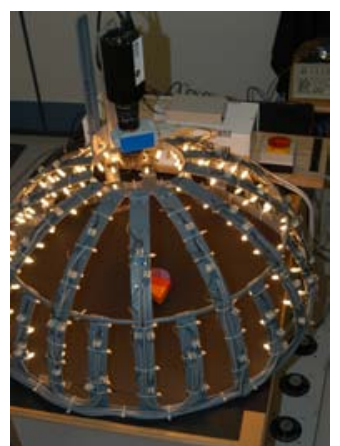

(a) Experimental set-up

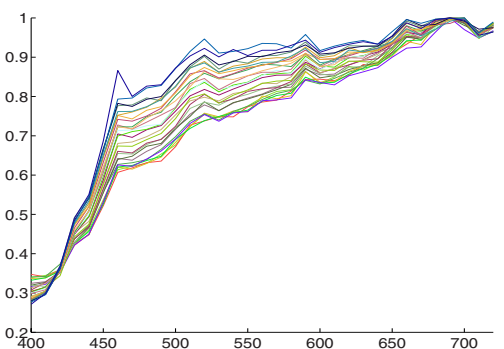

(b) Normalized Illumination spectra

Fig. 1. (a) Experimental set-up with hemispherical illumination and 3 phase potenciometers, (b) Spectra of the 26 illumination levels

distribution of the lamps inside of the hemisphere avoids flickering effects, due to the AC current. By adjusting the voltage level of the illumination chamber power supply we can change the spectral power distribution of the illuminant. The experimental set-up is shown in Figure 1 (a).

\section{Illumination Transformation Estimation}

\subsection{Change in Illumination of the Experimental Set-Up}

We change the power supply of the illumination uniformly over the whole range to get 26 different illumination levels and for each illuminant we capture an image of a perfect reflectance diffuser object (spectralon) to serve as a spectral descriptor of the light source. In Figure 1 b) the spectral power distribution of the illuminants is shown. They are normalized to 1 dividing each one by its own maximum.

We first characterize the properties of light sources. This is done using the following methods:

Colour Temperature: It is defined as the temperature in Kelvins at which a heated black-body radiator matches the hue of a lamp [16]

Colour Rendering: Defined as a value in the interval $[0-100]$ which measures the effect of a light source on the colour appearance of objects in comparison with their colour appearance under a reference illuminant (see [3]).

Colour Difference in the CIELAB and CIELUV Colour Spaces: These Coordinate systems are derived from properties of human color vision in which euclidean distance corresponds to perceptual difference (for a description, see [16]).

Figure 2 shows the changes in Colour Temperature, Colour Rendering, the chromaticity measured as $\left(a^{*}, b^{*}\right)$ vectors in the CIELAB system (see Section 4.2 for more details on CIELAB conversion), and the change in Lightness vs the chromaticity $C_{a b}^{*}$ for the 26 illuminants. The $26 t h$ illuminant (with highest $L^{*}$ value) is taken as reference. For the calculation of the CIELAB colour difference, the procedure to manage the 
white point explained in section 4.2 is used. From Figures 2 a) and (b) we can see that the illuminants correspond to different Colour Temperatures and that they are similar to the corresponding Black Body Radiators (i.e., Colour Rendering values are very close to 100). Figures 2 (c) and (d) show significant variation both in $L^{*}$ and Chroma values. Figure 3 shows an important colour difference measured in CIELAB and CIELUV colour spaces for the illumination changes.

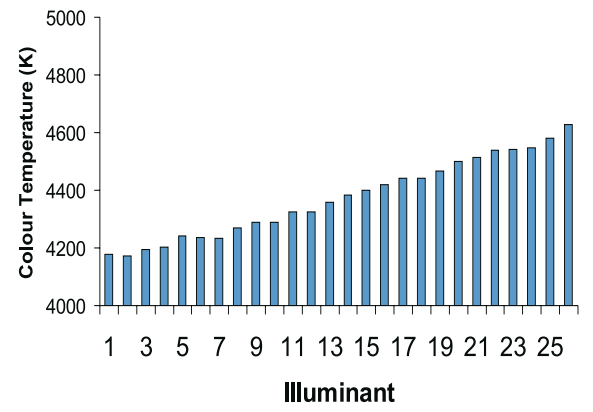

(a) Colour Temperature

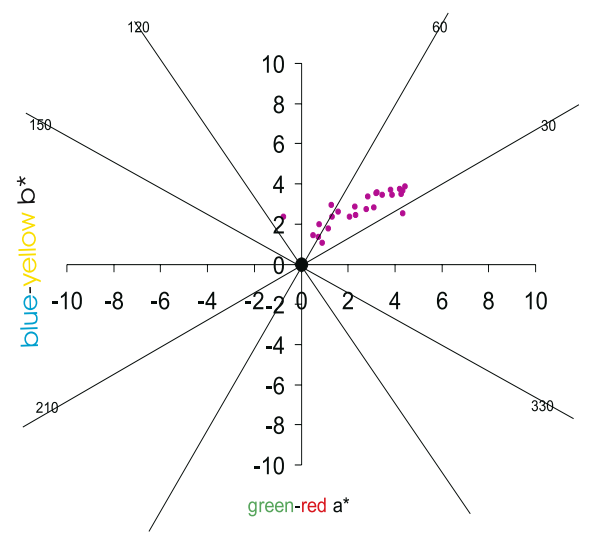

(c) $a^{*}$ vs $b^{*}$

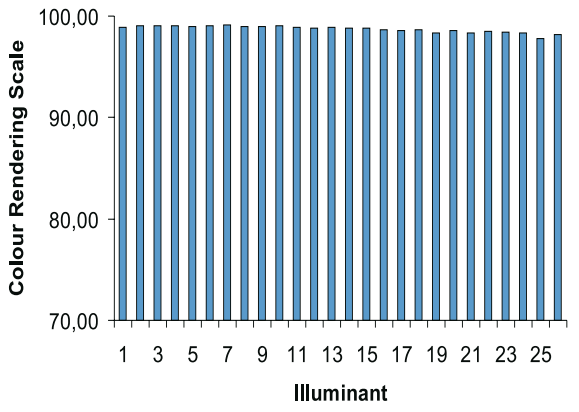

(b) Colour Rendering

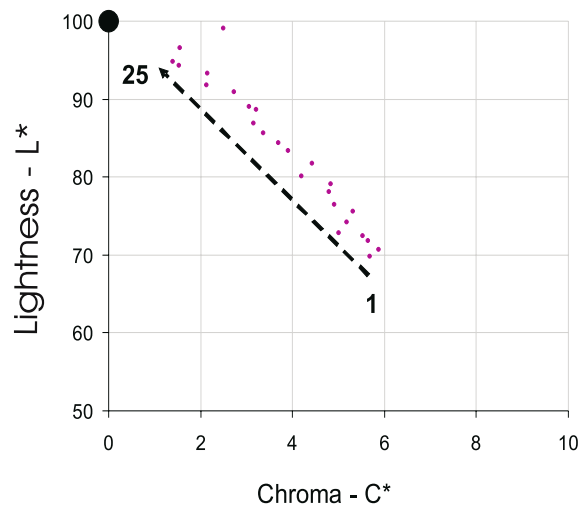

(d) Lightness vs $C_{a b}^{*}$

Fig. 2. Analysis of the 26 Illumination spectra

\subsection{Assessment of the Compensation}

We take a series of 26 images of 4 wooden geometric objects (denoted as Image 1 to Image 26), together with the illuminants of Section 4.1 and apply the affine models to Image $k, k=2, \ldots, 26$ so they are as similar as possible to Image 1 (the reference image) after the affine transformation (where the ordering in $k$ is such that Image 26 corresponds to the illumination in Section 4.1 with highest $L^{*}$ value). We use the $C I E L A B$ colour space [11] to assess their compensation performance, converting the 


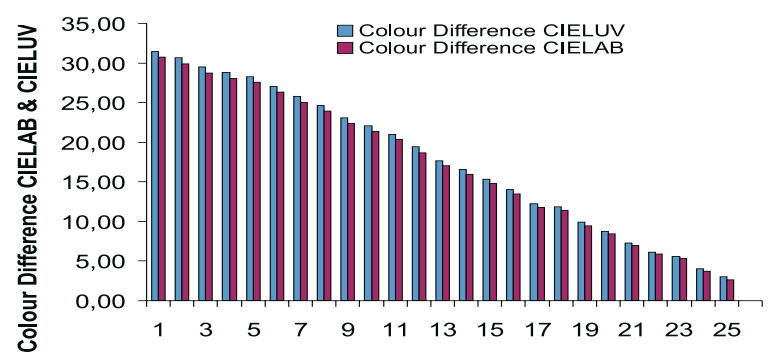

Fig. 3. $\Delta E_{a b}$ and $\Delta E_{u v}$ colour difference values between Illuminant $k(k=1, \ldots, 26)$ and Illuminant 26 , taken as reference

spectral curves of each image of the geometrical objects using the conventional formulae to change from spectra to the $X Y Z$ colour space [11]:

$$
X \propto \sum_{\lambda} P(\lambda) R(\lambda) \bar{x}(\lambda), \quad Y \propto \sum_{\lambda} P(\lambda) R(\lambda) \bar{y}(\lambda), \quad Z \propto \sum_{\lambda} P(\lambda) R(\lambda) \bar{z}(\lambda)
$$

where $P(\lambda)$ is the Power Distribution of the Illuminant, $R(\lambda)$ is the reflectance spectrum of the object and the product $P(\lambda) \cdot R(\lambda)$ is the signal arriving at the camera. $[\bar{x}(\lambda), \bar{y}(\lambda), \bar{z}(\lambda)]$ are the Colour Matching Functions of the $10^{\circ} 1964$ CIE Supplementary Standard Observer [11]. The white point change between illuminations $\left(X_{n i}, Y_{n i}, Z_{n i}\right), i=1, \ldots 26$, is obtained evaluating the $X Y Z$ coordinates of the images of the spectralon for each illuminant, and normalizing the $Y$ value of the highest illuminant to 100 . The rest of the $\left(X_{n i}, Y_{n i}, Z_{n i}\right)$ values are changed accordingly:

$$
X_{n i} \leftarrow \frac{X_{n i}}{Y_{n 26}} \cdot 100, \quad Y_{n i} \leftarrow \frac{Y_{n i}}{Y_{n 26}} \cdot 100, \quad Z_{n i} \leftarrow \frac{Z_{n i}}{Y_{n 26}} \cdot 100
$$

This normalization constant is used for any CIELAB colour difference.

\subsection{Model Evaluation in the Original Space}

In Figure 4 we can see the mean $\Delta E_{a b}$ Colour Difference for the comparison between Image 1 and the rest for the 3 models (general, particular and diagonal). The application of the first 2 models decreases $\Delta E_{a b}$ substantially, but this is not the case for the diagonal model.

In Figure 5 we show that the general affine transformation model gives better $\Delta E_{a b}$ results than the particular affine model for low illumination differences between images. As the difference gets higher, both Models tend to give similar results, though the particular model tends to oscillate more. We also generate simulated $R G B$ images before and after compensation using the $33 \times 3$ transformation matrix of a commercial camera obtained following the procedure described in [14]. In Figure 6] we see the Simulated Images 1, 26, and the change from 26 to 1 using the general affine model. 


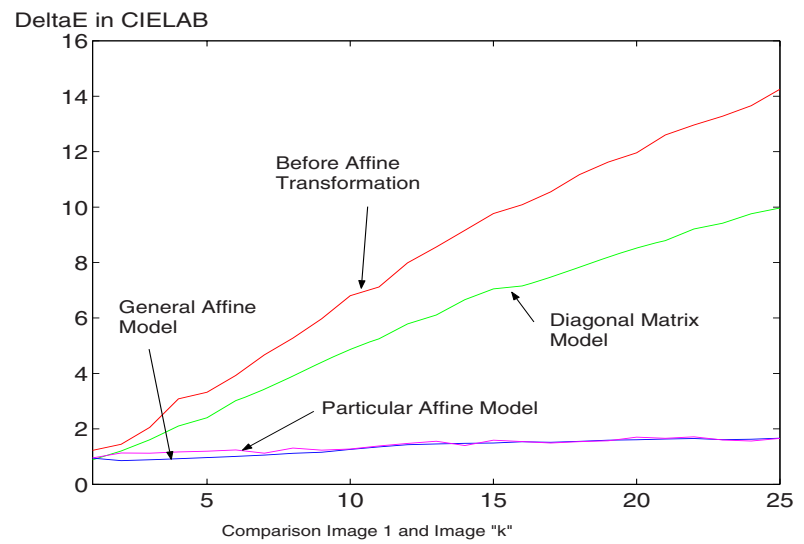

Fig. 4. Comparison mean $\Delta E_{a b}$ value before and after compensation using the general, particular and diagonal affine transformation models in the 33 dimensional space between Image 1 and Image $k$ with $k=2, \ldots, 26$

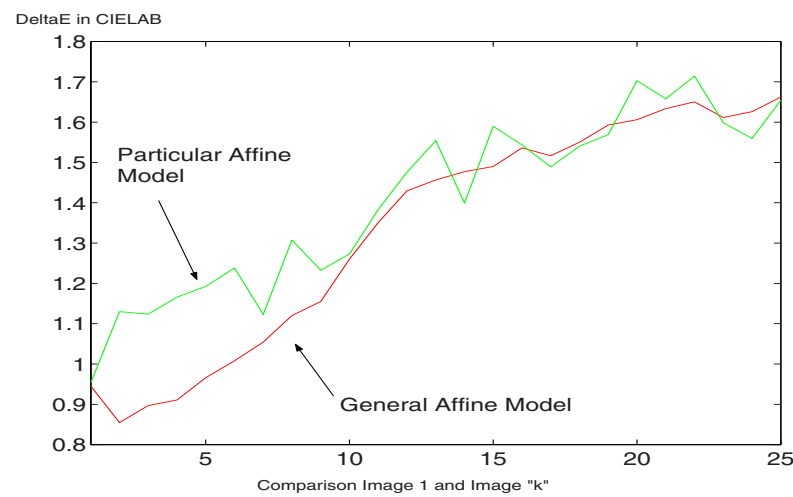

Fig. 5. Zoomed version Comparison mean $\Delta E_{a b}$ value before and after compensation using both affine transformation models in the 33 dimensional space

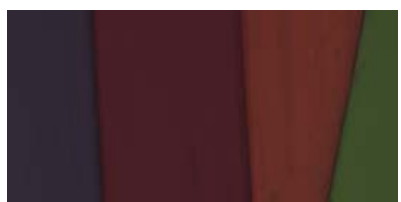

(a) $R G B 1$

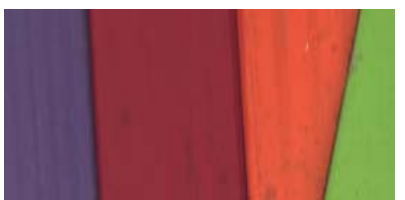

(b) $R G B 26$

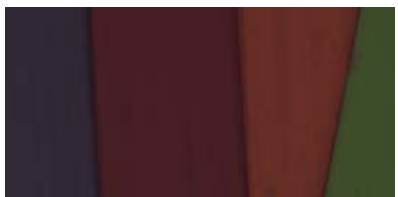

(c) $R G B 26 \rightarrow 1$

Fig. 6. Simulated $R G B$ Images created with the sensitivity curves of a Nikon camera 


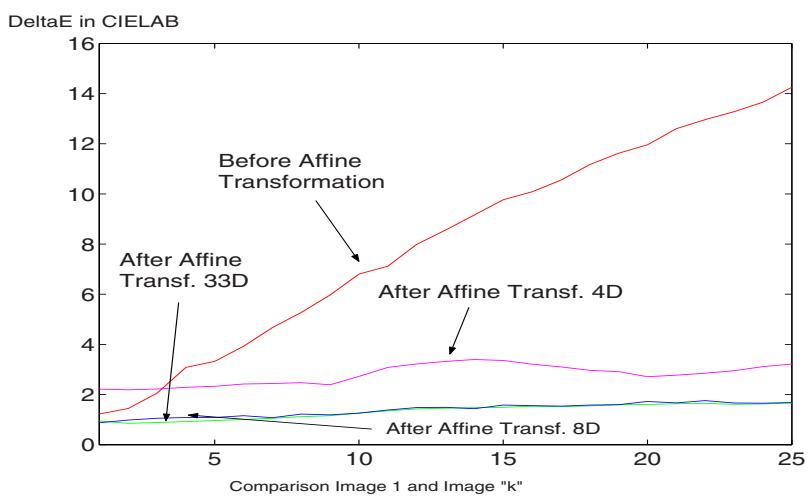

Fig. 7. Mean $\Delta E_{a b}$ value using the general affine transformation model for a 4, 8 and 33 dimensional space

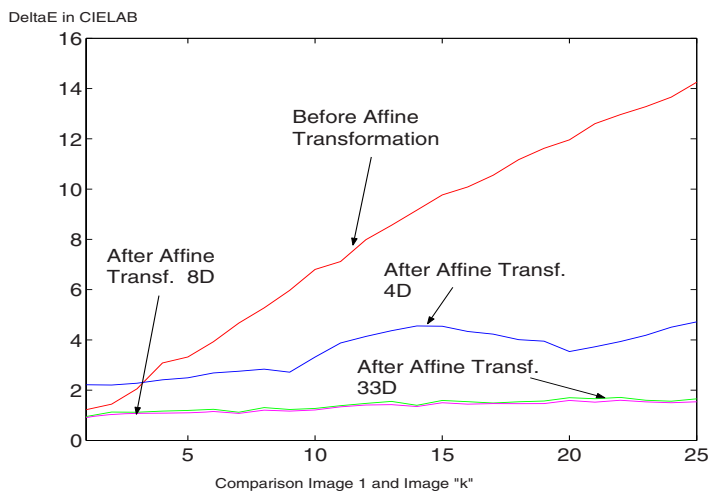

Fig. 8. Mean $\Delta E_{a b}$ value using the particular affine transformation model for a 4,8 and 33 dimensional space

\subsection{Model Evaluation in a Low-Dimensional Space}

We focus on the first two types of models. We apply the Singular Value Decomposition transform to a random selection of the $5 \%$ of the whole amount of pixels of the 26 multispectral images acquired under different illuminations. We make a selection of the first 4 and 8 eigenvectors ordered by their corresponding eigenvalues, and project the original images in the 33 dimensional space to these lower dimensional spaces using the following matrix projection formula:

$$
\mathbf{X}_{j D}=\mathbf{X}_{33 D} \cdot \mathbf{P}_{33 \rightarrow j},
$$

where $j=4,8$. We apply both affine transformation models in this lower dimensional space, and recover then the transformed data in the original space using:

$$
\mathbf{X}_{c h, 33 D}=\mathbf{X}_{c h, 6 D} \cdot \mathbf{P}_{33 \rightarrow j}^{t},
$$




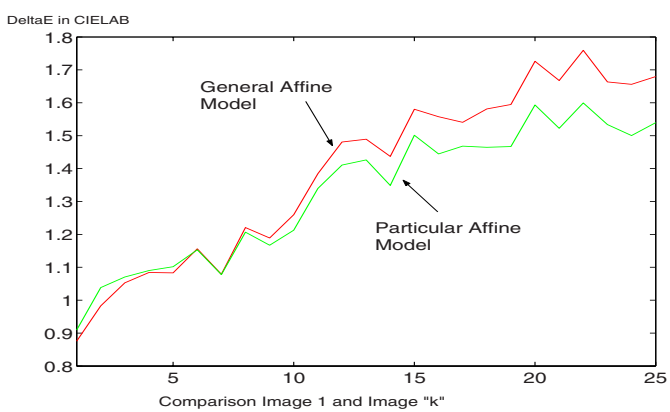

Fig. 9. Zoomed version comparison mean $\Delta E_{a b}$ value for the general and particular affine transformation models in an 8 dimensional space

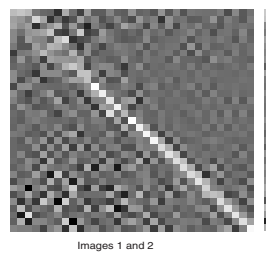

(a) $b_{i j} 2 \rightarrow 1$

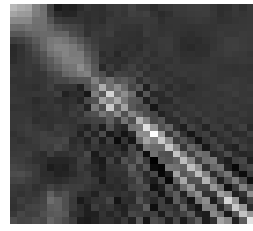

Images 1 and 2

(c) $a_{i j} 2 \rightarrow 1$

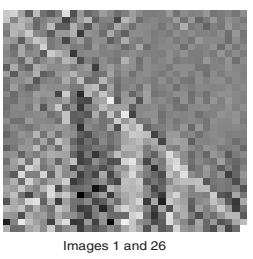

(b) $b_{i j} 26 \rightarrow 1$

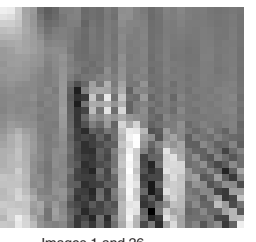

(d) $a_{i j} 26 \rightarrow 1$

Fig. 10. Grey-scale image general and particular models matrix elements in the original space

We evaluate the models in the same way as in Section 4.3 . Figures 7 and 8 show that we can work in an 8 dimensional space and obtain the same compensation performance results as in the original 33 dimensional space, for both models. In Figure 9 we show that for low illumination changes the general affine model gives better results than the particular affine model in an 8 dimensional space. Finally, in Figure 10 we present 2 examples of the matrix elements values of the models to analyze their distribution in the 33 dimensional space. The main diagonal and other parts of the matrices contribute. There is then an interaction among channels due to some internal device processes.

\section{Conclusion}

In this paper we showed that the affine model used by Heikkila et al in [10] for the analysis of rigid body movements can be applied to the compensation of illumination 
changes. This model gives better results in general than the particular affine model in the original 33 dimensional space. The analysis of illumination changes as affine transformations opens the door to the development of invariant representations of images.

\section{Acknowledgments}

This work has been partially funded by the Ministry of Education and Science of the Spanish Government through the DATASAT project $(E S P-2005-00724-C 05-$ C05). Pedro Latorre Carmona is a Juan de la Cierva Programme researcher (Ministry of Education and Science). The authors also thank Prof. Verdu for many useful discussions, and for the variability analysis of the illumination source.

\section{References}

1. Barnard, K., Finlayson, G., Funt, B.: Color constancy for scenes with varying illumination. Computer Vision and Image Understanding 65, 311-321 (1997)

2. Begelfor, E., Werman, M.: Affine invariance revisited. In: IEEE Conf. on Computer Vision and Pat. Rec. vol. 2, pp. 2087-2094 (2006)

3. Method of measuring and specifying colour rendering properties of light sources. CIETechnical Report 13.3 (1995)

4. Finlayson, G.D., Drew, M.S., Funt, B.V.: Spectral sharpening: sensor transformations for improved color constancy. Journal of the Opt. Soc. of America, A. 11, 1553-1563 (1994)

5. Finlayson, G., Chatterjee, S.S., Funt, B.V.: Color Angular Indexing. In: Buxton, B.F., Cipolla, R. (eds.) ECCV 1996. LNCS, vol. 1065, pp. 16-27. Springer, Heidelberg (1996)

6. Finlayson, G.D., Hordley, S.D., Xu, R.: Convex programming colour constancy with a diagonal-offset model. In: IEEE Int. Conf. on Image Processing vol. 3, pp. 948-951 (2005)

7. Healey, G., Slater, D.: Global color constancy: recognition of objects by use of illuminationinvariant properties of color distributions. Journal of the Opt. Soc. of America, A. 11, 3003 3010 (1994)

8. Healey, G., Slater, D.: Computing illumination-invariant descriptors of spatially filtered color image regions. IEEE Trans. on Image Proc. 6, 1002-1013 (1997)

9. Healey, G., Jain, A.: Retrieving multispectral satellite images using physics-based invariant representations. IEEE Trans. on Pat. Analysis and Mach. Intel. 18, 842-848 (1996)

10. Heikkilä, J.: Pattern Matching with Affine Moment Descriptors. Pattern Recognition 37, 1825-1834 (2004)

11. Hunt, R.W.G.: Measuring Colour, Fountain Press (1998)

12. Lenz, R., Tran, L.V., Meer, P.: Moment based normalization of color images, IEEE 3rd Workshop on Multimedia Signal Processing, pp. 103-108 (1998)

13. Schonemann, P.H.: A generalized solution of the orthogonal Procrustes problem. Psychometrika 31, 1-10 (1966)

14. Solli, M., Andersson, M., Lenz, R., Kruse, B.: Color measurements with a consumer digital camera using spectral estimation techniques. In: Kalviainen, H., Parkkinen, J., Kaarna, A. (eds.) SCIA 2005. LNCS, vol. 3540, pp. 105-114. Springer, Heidelberg (2005)

15. Sprinzak, J., Werman, M.: Affine Point Matching. Pat. Rec. Letters 15, 337-339 (1994)

16. Wyszecki, G., Stiles, W.S.: Color Science: concepts and methods, quantitative data and formulae, Wiley Classics Library (2000) 